\title{
Análisis estadísticu de les distancies prosódiques ente los dialeutos del asturianu / Statistical analysis of prosodic distances between the Asturian dialects
}

\author{
PAOLO Roseano \\ UNIVERSITAT DE BARCELONA \\ UNIVERSITY OF SOUTH AFRICA
}

\begin{abstract}
RESUME: A lo llargo de los caberos quince años la prosodia del asturianu foi oxetu d'estudiu dientro del marcu del proyeutu AMPER-ASTUR, destacándose ente los sos resultaos la clasificación de les variedaes entonatives d'esta llingua. Esti trabayu pretende analizar los datos prosódicos recoyíos n'AMPER-ASTUR emplegando ProDis, un programa dialeutométricu, col envís de validar les clasificaciones propuestes enantes. Los resultaos del procesamientu estadísticu de los datos amuesen que les declaratives neutres nun dexen trazar llendes xeoprosódiques nel dominiu llingüísticu asturianu, tal y como afiten estudios anteriores. L'análisis de les interrogatives absolutes, per otru llau, empobina a la conclusión de qu'Asturies apaez xebrada en dos fasteres principales: una centro-occidental (carauterizada por patrones interrogativos que finalicen con un tonu baxu) y una oriental (na que les entrugues totales presenten un cuerpu altu y un final circunflexu). Los resultaos tamién apunten a que, pa una clasificación más completa y detallada de la variación diatópica de la prosodia asturiana, fadrá falta en futuros estudios llevar a cabu una comparanza coles variedaes romániques averaes.
\end{abstract}

Pallabres clave: prosodia, dialeutometría, asturianu, AMPER-ASTUR, ProDis.

AbSTRACT: Over the past fifteen years, Asturian prosody has been studied in the frame of the AMPER-ASTUR project. One of its main results was the classification of the intonation varieties of this language. This paper analyses the prosodic data collected in the project using ProDis, a dialectometric tool, in order to validate the mentioned classification. The results of the statistical analysis show that in the case of neutral declaratives, it is not possible to determine geoprosodic boundaries in the Asturian linguistic domain, as suggested by previous studies. As for absolute interrogatives, the analysis leads to the conclusion that two different areas can be found in Asturies. The central-western part is characterized by interrogative patterns ending in a low tone, while in the eastern area, absolute questions have a high tone ending with a circumflex intonation. The results also indicate that further studies including a comparison with some close Romance varieties will allow to produce a more complete and detailed classification of the diatopic variation of Asturian prosody.

Keywords: prosody, dialectometry, Asturian, AMPER-ASTUR, ProDis. 


\section{INTRODUCCIÓN}

La prosodia de les llingües romániques foi oxetu d'estudiu dende destremaes perspeutives llinguístiques. Dende'l puntu de vista dialeutolóxicu, ún de los retos a los que s'enfrentó la comunidá científica ye'l de documentar los patrones entonativos de les distintes variedaes del ámbitu románicu, oxetivu que ta algamándose anguaño gracies a la creación de dos bases de datos: l'Atlas Multimedia de Prosodia del Espacio Románico (Contini 1992, Romano 2001, Contini et al. 2003, Romano et al. 2005) y l'Interactive Atlas of Romance Intonation (Prieto, Roseano y Borràs-Comes 2010-2014).

L'estudiu de la prosodia del asturianu afondóse nel marcu del Atlas Multimedia de Prosodia del Espacio Románico en rellación con dos modalidaes oracionales: la declarativa neutra y la interrogativa total neutra. A partir de los datos recoyíos nesi proyeutu, non sólo se describieron los patrones entonativos de los distintos dialeutos de la llingua asturiana y de les variedaes cercanes sinón que tamién se propunxeron delles clasificaciones dialeutales que se resumen nel apartáu d'esti trabayu dedicáu a «Les descripciones de la prosodia del asturianu». L'oxetivu principal d'esti artículu ye'l de validar les clasificaciones dialeutales propuestes anteriormente, emplegando'l conxuntu de téuniques d'análisis estadísticu que se conocen como dialeutometría entonativa o prosodometría, presentándose les carauterístiques xenerales d'esta disciplina nel epígrafe «La dialeutometría entonativa».

Acordies con esti envís, el trabayu estructúrase en tres partes principales. La primera d'elles presenta l'estáu de la cuestión na estaya de los estudios sobro la prosodia del asturianu (ufiertando una descripción de los patrones entonativos principales) y un resume de los avances de la dialeutometría entonativa nes caberes décades. La segunda parte dedícase a aspeutos metodolóxicos y nella, arriendes de describise'l corpus emplegáu, sintetícense les carauterístiques xenerales del programa d'análisis dialeutométrico manexáu (ProDis). Darréu, nel apartáu de resultaos, preséntense los análisis de los datos entonativos del asturianu que se llevaron a dialeutometrización. Ponen puntu al trabayu unes consideraciones conclusives y plantéguense unes llinies de trabayu futures.

\section{Les descripciones de la prosodia del asturianu}

L'estudiu de la prosodia de les variedaes llingüístiques d'Asturies (l'asturianu, el gallego-asturianu y el castellanu) fíxose nel marcu del proyeutu AMPER-ASTUR que, de magar l'añu 2004 y baxo la direición de la Dra. Muñiz Cachón del Llaboratoriu de Fonética de la Universidá d'Uviéu, 
permitió la creación d'un atles online de prosodia d'Asturies, lo mesmo que l'asoleyamientu d'una pergranible cantidá de publicaciones. Ente elles, dalgunes destáquense por ser descripciones detallaes de zones xeoprosódiques concretes (como, por exemplu, López Bobo et al. 2005 pa Uviéu y Mieres; López Bobo et al. 2008 pa la fastera occidental; Muñiz Cachón et al. 20062007-2008 pal gallego-asturianu; Muñiz Cachón y Alvarellos Pedrero 2008 pa la fastera oriental) o por ufrir una visión de conxuntu de la entonación de les variedaes romániques d'Asturies (Muñiz Cachón et al. 2010; Alvarellos Pedrero et al. 2011; Muñiz Cachón 2013).

Los estudios anteriores coinciden en describir un únicu patrón pa les declaratives neutres del asturianu, con variaciones locales perpoco pronunciaes. L'esquema entonativu d'esa modalidá oracional coincide tamién, por alzao, col que s'alvierte n'otres variedades romániques de la península ibérica, como'l castellanu y el catalán. Carauterízase per picos tonales asitiaos nes sílabes postóniques de les primeres pallabres acentuaes del enunciáu y, na parte terminal, per una baxada adulces que va dende l'últimu picu tonal hasta'l final del enunciáu. El patrón en cuestión ta exemplificáu na figura 1, que se refier al enunciáu «El paquete llevaba pelotes» pronunciáu por una informante d'Uviéu.

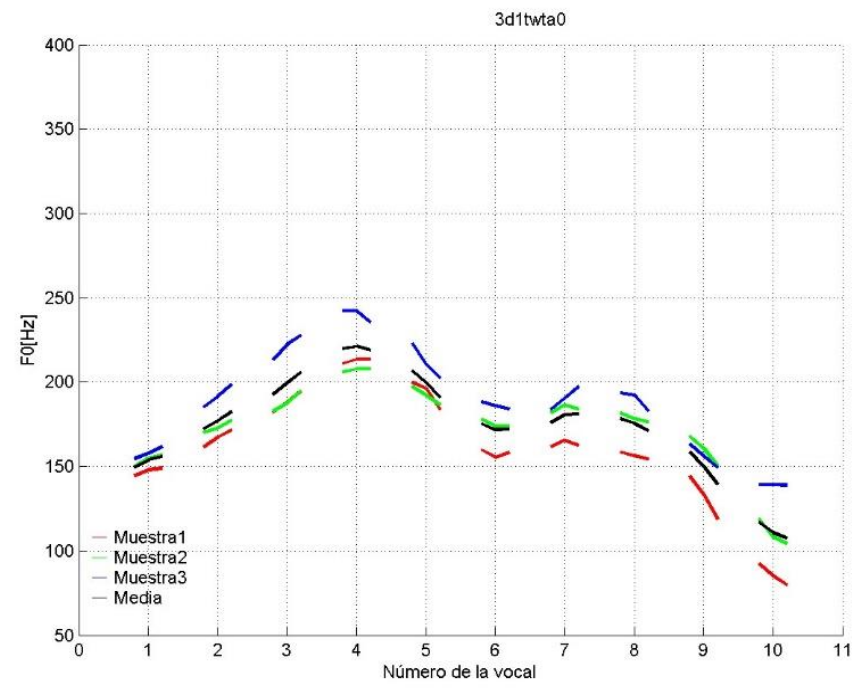

Figura 1. Contornu entonativu de les tres repeticiones y de la media de la declarativa «El paquete llevaba pelotes» pronunciada por una informante d'Uviéu (fonte: Muñiz Cachón et al. 2019)

La entonación de les interrogatives totales presenta una variación xeográfica más destacada. Na parte occidental d'Asturies apaecen patrones que se denomaron n'inglés hat pattern, porque presenten un asemeyu cola silueta d'un sombreru vistu de perfil. Carauterícense por un ascensu tonal inicial, un cuerpu central altu y una 
rápida baxada final, acordies como vemos n'El Franco (figura 2) o Tinéu (figura 3). Ye interesante recordar que'l patrón en cuestión ufre un claru asemeyu col esquema entonativu de les entrugues absolutes del gallegu (Fernández Rei y de Castro Moutinho 2016; Fernández Rei 2016).

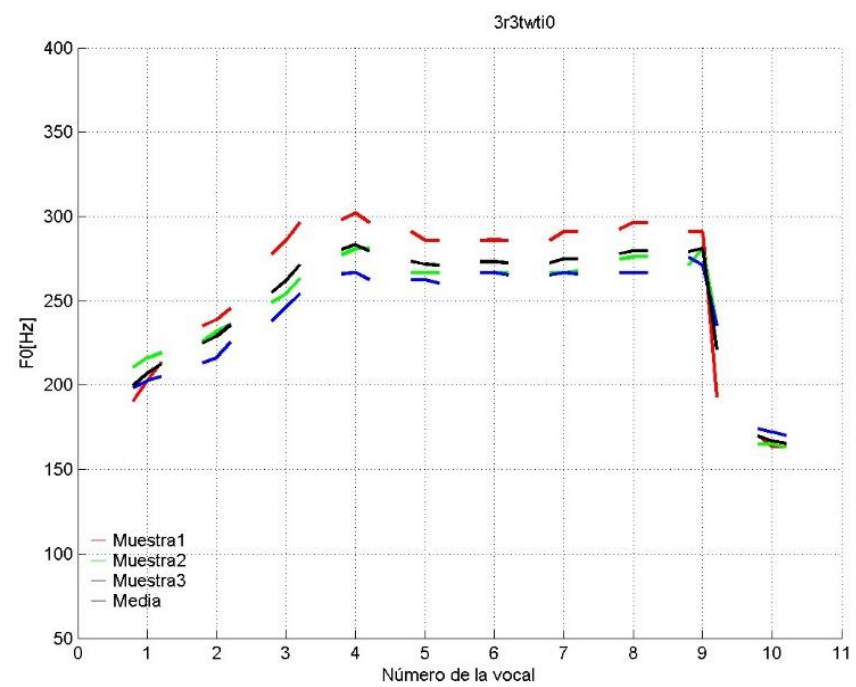

Figura 2. Contornu entonativu de les tres repeticiones y de la media de la interrogativa absoluta « ¿A rapaza prepara patacas?» pronunciada por una informante d'El Franco (fonte: Muñiz Cachón et al. 2019)

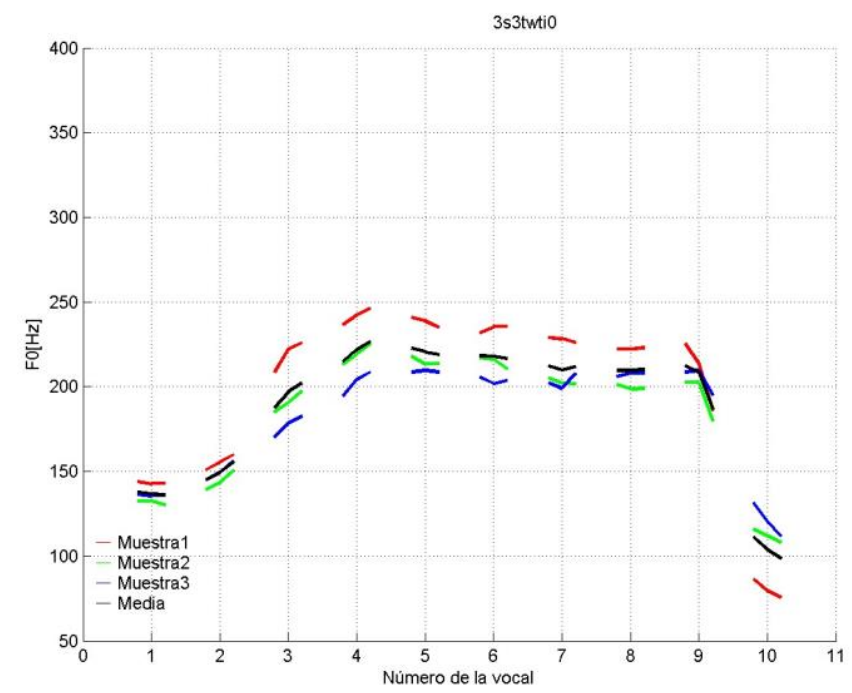

Figura 3. Contornu entonativu de les tres repeticiones y de la media de la interrogativa absoluta « ¿La rapaza cocina patacas?» pronunciada por una informante de Tinéu (fonte: Muñiz Cachón et al. 2019) 
Na parte central del dominiu llinguísticu la entonación de les interrogatives sigue siendo descendente al final, pero -al contrariu que nel occidente- presenta un valle nel cuerpu del enunciáu, tal y como s'alvierte nos datos d'Uviéu (figura 4) o de Mieres (figura 5). Nesti últimu llugar ha destacase que la prosodia de les interrogatives ta carauterizada por un fenómenu que nun ye de calter entonativu sinón de calter duracional y que consiste nel allargamientu de les vocales finales del enunciáu (pa una descripción en detalle ver López Bobo et al. 2005).

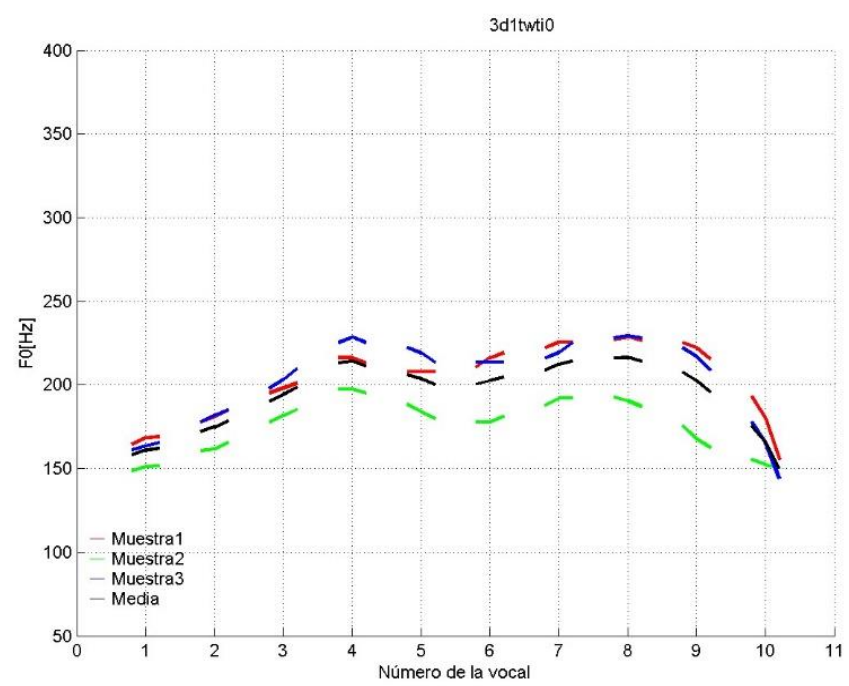

Figura 4. Contornu entonativu de les tres repeticiones y de la media de la interrogativa absoluta $\ll_{\text {¿El }}$ paquete llevaba pelotes?» pronunciada por una informante d'Uviéu (fonte: Muñiz Cachón et al. 2019)

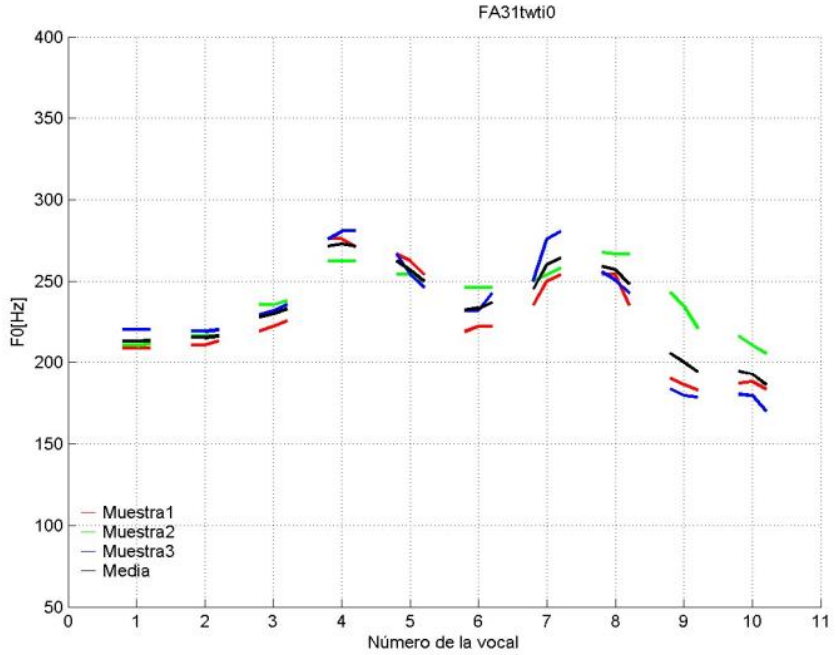

Figura 5. Contornu entonativu de les tres repeticiones y de la media de la interrogativa absoluta « ¿El paquete llevaba pelotes?» pronunciada por una informante de Mieres (fonte: Muñiz Cachón et al. 2019) 
Finalmente, nel oriente d'Asturies (en concretu en Ribeseya) apaez un patrón de calter diferente (figura 6), carauterizáu por un final que presenta una sílaba tónica realizada nun tonu altu, siguida d'un movimientu ascendentedescendente na parte final (ensin que'l F0 vuelva al nivel baxu que presentaba al entamu del enunciáu). Nesti sen, la entonación interrogativa de Ribeseya allóñase de les anteriores, que comparten un descensu final qu'algama hasta la estaya más baxa de la tesitura de los informantes.

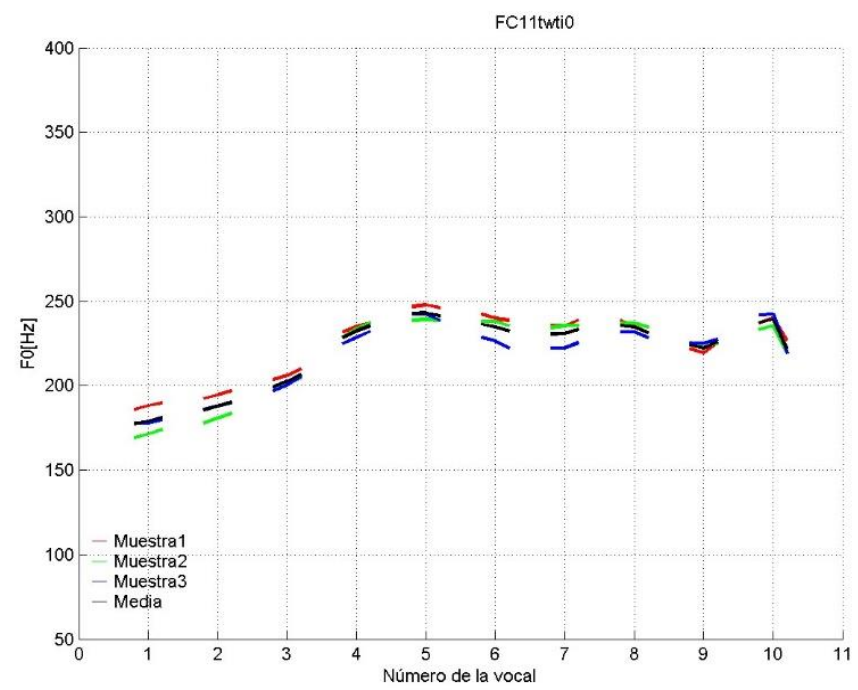

Figura 6. Contornu entonativu de les tres repeticiones y de la media de la interrogativa absoluta « ¿La rapaza cocina patates?» pronunciada por una informante de Ribeseya (fonte: Muñiz Cachón et al. 2019)

En definitiva, los estudios sobre la prosodia del asturianu (y más en particular Muñiz Cachón et al. 2010; Alvarellos Pedrero et al. 2011; Muñiz Cachón 2013) afirmen que la entonación declarativa nun dexa afitar nenguna dixebra dialeutal, mentanto que la entonación interrogativa suxer la presencia de tres zones: dos d'elles carauterizaes por un final baxu y una carauterizada por un descensu final de F0 que nun algama hasta la llinia de base del informante.

\section{La dialeutometría entonativa}

La esmolición pola necesidá de midir de mou oxetivu les distancies ente variedaes llingüístiques surdió nos años 70 del sieglu XX y empobinó a la nacencia de la disciplina conocida como Dialeutometría (ver, ente otros, Séguy 1971 y Goebl 1982), que consiste n'aplicar téuniques d'análisis estadísticu a bases de datos perestenses (por un regular atles llingüísticos con información de calter léxicu y fonético-segmental). Según se tien destacao 
apocayá en dalgún trabayu (Roseano 2016), col surdimientu, delles décadas dempués, de los atles llingüísticos dedicaos a los aspeutos suprasegmentales, plantegóse la necesidá de disponer de preseos d'análisis que llograren someter a análisis dialeutométricu los nuevos datos. Cuando los entonólogos quixeron aplicar téuniques dialeutométriques a les sos bases de datos (que pal casu del Atlas Multimedia de Prosodia del Espacio Románico inxeríen namái datos numéricos), alcontráronse con que nun podíen mandase de preseos acionaos, porque los programes d'análisis dialeutométricu qu'existíen ${ }^{1}$ taben pensaos p'analizar sólo (o casique sólo) datos alfabéticos. Por esti motivu, ún de los oxetivos metodolóxicos que más priesa corría yera'l de crear un preséu dialeutométricu axeitáu, que fuere quien pa llevar a cabu les dos operaciones básiques de los preseos dialeutométricos tradicionales: 1) crear una matriz de distancies numbériques a partir de los datos del atles y 2) tresformar la matriz nuna (nunes) representación (representaciones) gráfica (gráfiques) d'interpretación fácil que permitieren al dialeutólogu visualizar de mou inmediatu los resultaos del análisis estadísticu.

La dialeutometría entonativa (denomada tamién Prosodometría) que surdió asina ye una disciplina con una historia relativamente curtia, que da principiu a finales de los años noventa. Los primeros entamos pa calcular la distancia ente dos curves entonatives débense a Hermes (1998a, 1998b). La so propuesta, sicasí, nun llegó a perfacese darréu na estaya dialeutolóxica. Hubo qu'aguardar dalgunos años pa qu'ello asocediere: ye a principios del sieglu XXI cuando entamen a aplicase les fórmules d'Hermes a bases de datos de variación diatópica de la entonación. Nesta primer fase'l centro impulsor de la dialeutometría entonativa ye'l grupu de trabayu que tien como puntu referencia'l Centre de Dialectologie de Grenoble. Más en concretu, ye colos trabayos d'investigación rellacionaos cola tesis doctoral de Romano (1999) cuando principia a esplorase la posibilidá d'aplicar téuniques dialeutométriques a la base de datos prosódicos que taba empezando a construise naquellos años: AMPER (Contini 1992; Contini et al. 2003).

Los dos programes que s'empleguen nesta primer fase afítense, básicamente, nes fórmules d'Hermes (1998a, 1998b) o n'adautaciones de les mesmes. El primeru d'ellos, conocíu como Amper StatDistances, foi creáu por Rillard y Lai en marcu del proyeutu AMPER (Rillard y Lai 2008; Moutinho et al. 2011) col envís de dialeutometrizar los datos numbéricos de la F0 de los enunciaos que se recueyen acordies cola metodoloxía del mesmu proyeutu. Esi aplicativu, a partir de los datos prosódicos que correspuenden a tres valores de F0 (en $\mathrm{Hz}$ ) en cada vocal del enunciáu (inicial, central y final), más un valor

${ }^{1}$ En concretu, Visual DialectoMetry (Haimerl 2006), Gabmap (Nerbonne, Colen, Gooskens, Kleiweg y Leinonen 2011) y DiaTech (Aurrekoetxea Fernández-Aguirre, Rubio, Ruiz y Sánchez 2013). 
d'intensidá en caúna d'elles, ufre una representación gráfica de los averamientos ente dialeutos y de les agrupaciones de los mesmos en forma de mapa EMD (figura 7) y de dendrograma (figura 8).

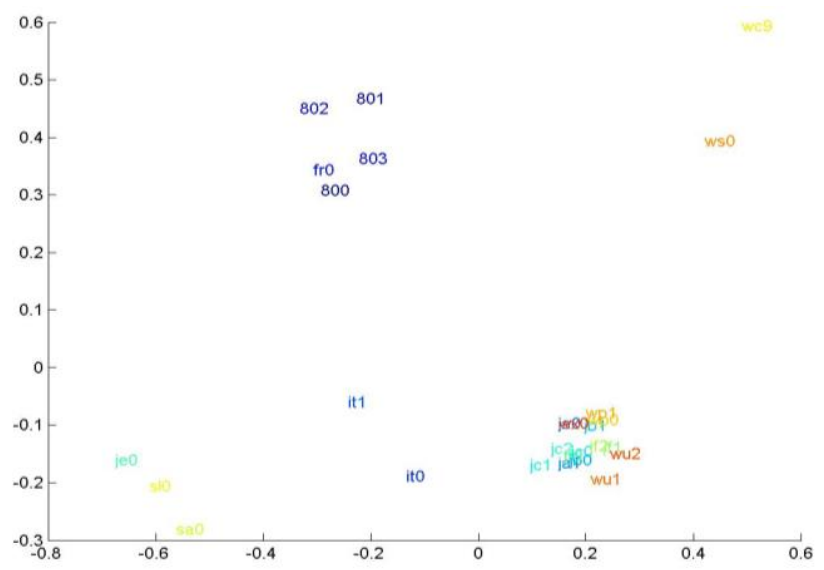

Figura 7. Mapa EMD que representa les distancies entonatives ente distintes variedaes romániques fechu con Amper StatDistances (fonte: Roseano 2012)

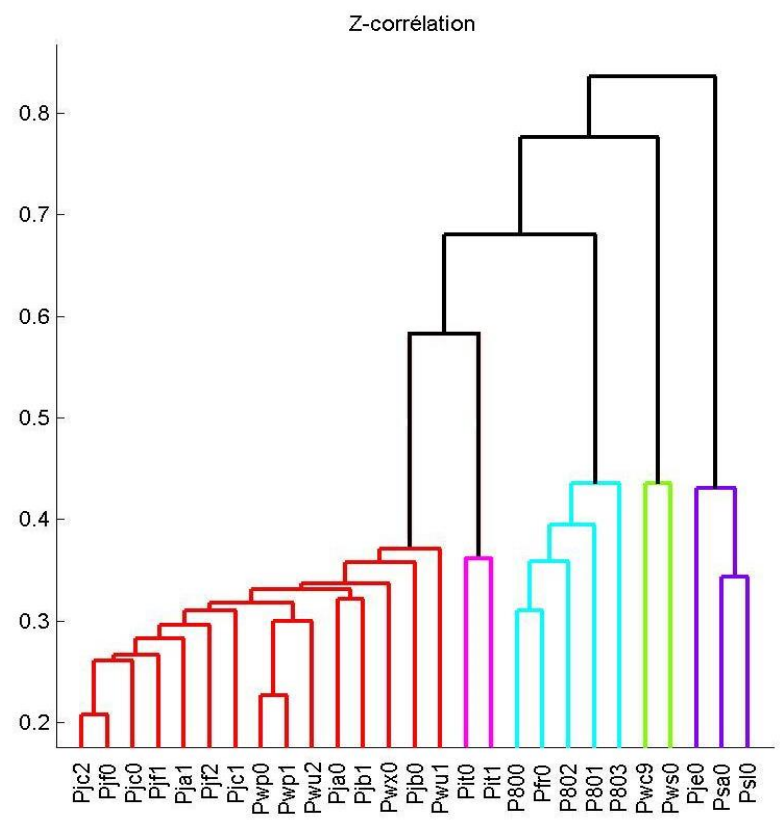

Figura 8. Dendrograma que representa les distancies entonatives ente distintes variedaes romániques fechu con Amper StatDistances (fonte: Roseano 2012) 
La torga principal d'Amper StatDistance asitiábase nel fechu de que nun permitía visualizar la matriz de distancies nin escoyer los métodos de conglomeración. Por esi motivu nel Laboratori de Fonètica de la Universitat de Barcelona ellaboróse una rutina, denomada Calcu-Dista, que foi quien pa iguar l'enzancu en cuestión. Esa rutina (Roseano, Fernández Planas, ElviraGarcía y Martínez Celdrán 2015) toma como base de los datos l'output del análisis numbéricu que suel llevase a cabu nel marcu AMPER y crea, per aciu d'un script de Praat, una base de datos nun formatu Excel que pue analizase con SPSS. Llueu de que los datos numbéricos en cuestión s'importen en SPSS, ye posible aplicar téuniques de clustering equivalentes a les que s'implementen na dialeutometría tradicional. La ventaya, en comparanza con Amper StatDistances, consiste nel fechu de que, faciéndolo d'esta miente, en SPSS ye posible seleicionar los algoritmos afayadizos y ufrir los indicadores estadísticos (como'l stress) que nel programa anterior quedaben tapecíos. El mesmu SPSS permitía llegar a una representación gráfica, en forma de dendrograma, de los resultaos del análisis dialeutométricu. A pesar de la mayor flexibilidá, la rutina del Laboratori de Fonètica qu'acaba presentase siguía plantegando dalgunos defeutos. El más importante d'ellos yera, de xuru, lo trabayoso de la mesma. De fechu, la ellaboración d'un análisis dialeutométricu con esos preseos implica una riestra d'operaciones informátiques (que nesti trabayu nun se detallen por motivos d'espaciu) que representaben un costu mui altu en términos de tiempu. Por esti motivu, nos años siguientes foron surdiendo rutines más cencielles.

L'añu que marca un finxu nesti camín ye'l de 2015, al presentase oficialmente dos preseos dialeutométricos ellaboraos de forma independiente en dos equipos rellacionaos col proyeutu AMPER: la Ferramenta de la Universidá de Santiago de Compostela (Martínez Calvo y Fernández Rei 2015) y ProDis (Elvira-García, Balocco, Fernández Planas, Roseano y Martínez Celdrán 2015), el nuevu programa dialeutométricu del Laboratori de Fonètica de la Universitat de Barcelona. Anque presenten diferencies ente ellos, los dos preseos respuenden a unes esixencies comunes y ufierten unes soluciones comparatibles. Per una parte, de fechu, proponíense axilizar l'análisis dialeutométricu de los datos entonativos conteníos na base de datos d'AMPER ufiertando un software únicu que permitiere llevar a cabu toles operaciones que, en fases anteriores, esixíen programes estremaos. D'otru llau, enanchaben l'abanicu de resultaos estadísticos que podíen algamase con SPSS y amestaben la posibilidá de representar los mesmos resultaos en mapes xeográficos. Amás, dirixíense dambos a un públicu que nun tien conocimientos de programación informática. Pa esti trabayu emplegóse ProDis, sobro'l que se volverá nel apartáu dedicáu al «Análisis dialeutométricu de los datos». 


\section{METOdOLOXíA}

\section{Corpus ya informantes}

El corpus d'esti estudiu consiste en 3.780 enunciaos que formen parte de la base de datos d'AMPER-ASTUR (Muñiz Cachón et al. 2019), la seición asturiana del Atlas Multimedia de Prosodia del Espacio Románico (Contini 1992; Contini et al. 2003; Romano et al. 2005). Los enunciaos correspuenden a declaratives neutres ya interrogatives absolutes neutres formaes por un suxetu, un verbu y un oxetu. El suxetu y l'oxetu puen ser palabres agudes, llanes y esdrúxules, mentanto que'l verbu siempre ye llanu. En cuantes a la so cadarma sintáutica, los enunciaos declarativos son SVO (como, por exemplu, «El paquete llevaba pelotes»). Lo mesmo'l suxetu que l'oxetu puen ser simples (como nel casu anterior) o dir acompañaos d'un elementu (axetivu o sintagma preposicional) que fai d'espansión (como, por exemplu, en «El paquete pequeñu llevaba pelotes»o «El paquete llevaba pelotes pequeñes»). $\mathrm{Pa}$ cada modalidá oracional, analizáronse tres repeticiones de nueve enunciaos (compuesta caúna una por una combinación diferente de palabres agudes, llanes y esdrúxules).

Los enunciaos emitiéronlos 10 informantes - homes y muyeres-, de 5 puntos d'encuesta (figura 9) asitiaos en zones destremaes del dominiu llingüísticu asturianu: El Franco na fastera de gallego-asturianu, Tinéu na del asturianu occidental, Uviéu na del asturianu central, Mieres en representación del asturianuo centro-sureñu, y Ribeseya pa la fastera del asturianu oriental.

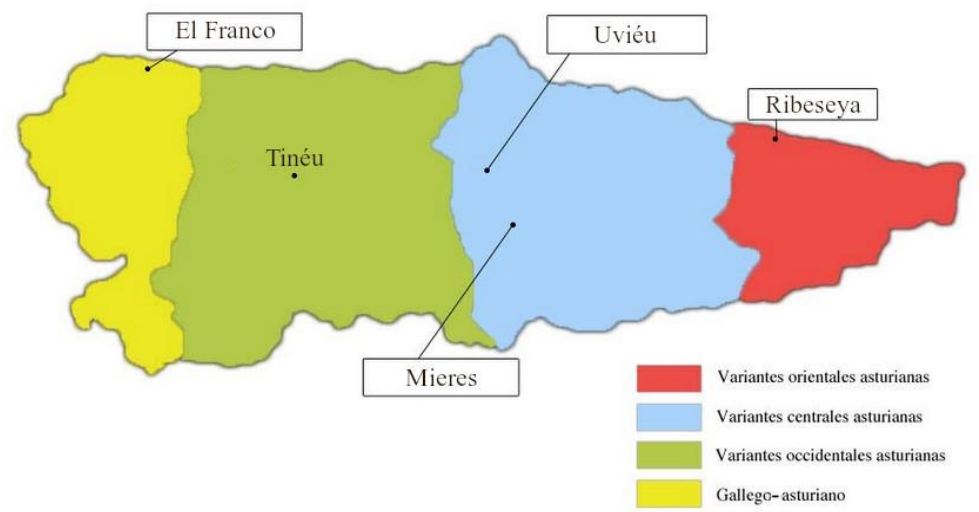

Figura 9. Mapa cola conseñación de los puntos d'encuesta (fonte: Muñiz Cachón et al. 2019) 


\section{Análisis acústicu de los datos}

L'análisis acústicu de los enunciaos grabaos llevóse a cabu per aciu d'Amper-2006 (figura 10), un programa creáu pol Llaboratoriu de Fonética de la Universidá d'Uviéu (López Bobo et al. 2007) dende unes rutines afitaes por Romano (Romano et al. 2005). Amper-2006 estrái pa cada vocal d'un enunciáu un valor de duración, unu d'intensidá y tres de $\mathrm{F} 0$ en $\mathrm{Hz}$ y en semitonos (F0 inicial, F0 central y F0 final). Los valores en cuestión almacénense n'archivos de testu que conformen l'input del programa de dialeutometría del que se detallen les carauterístiques nel apartáu siguiente. Amper-2006 tamién xenera gráficos que contienen les curves de F0 estilizaes de los enunciaos, como los que se presentaron nes figures 1 a 6 .

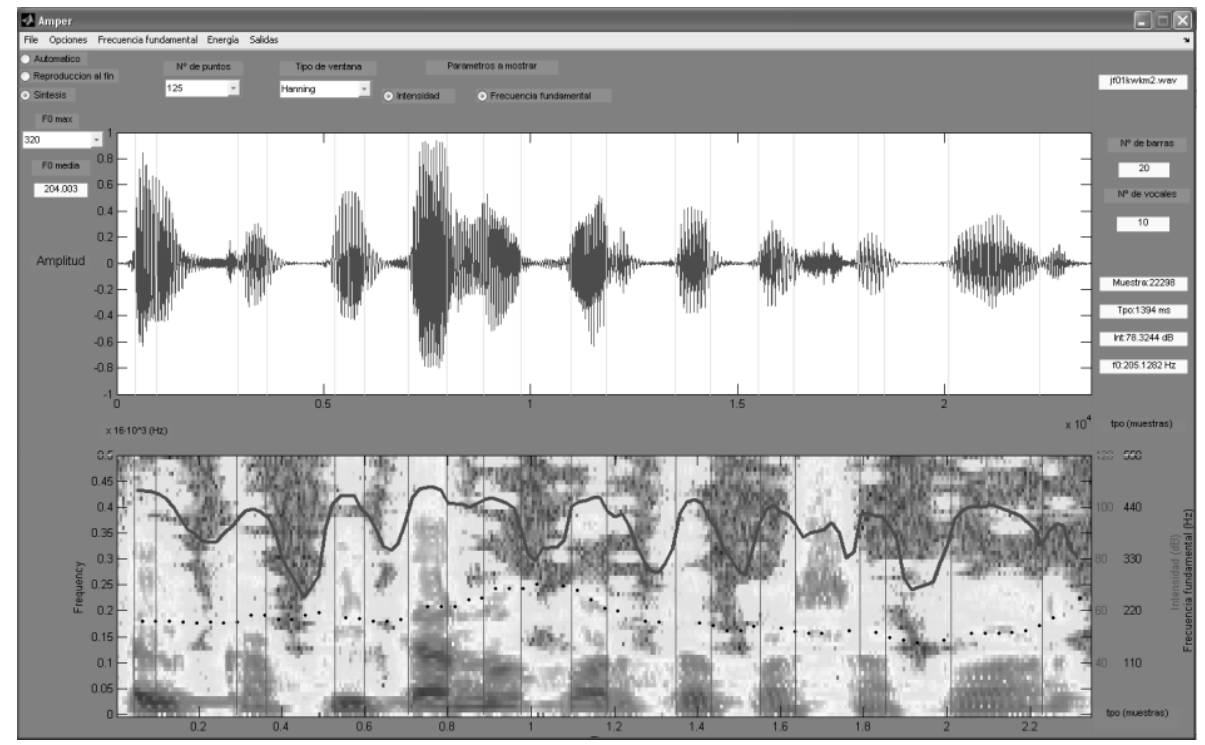

Figura 10. Oscilograma, espeutrograma y curva de F0 d'un enunciáu analizáu con Amper-2006

\section{Análisis dialeutométricu de los datos}

El programa d'análisis dialeutométricu emplegáu nesti trabayu ye ProDis (Elvira-García et al. 2018). L'input de ProDis son los valores numbéricos sacaos por Amper-2006 y que constituyen, dende'l puntu de vista matemáticu, un vector. ProDis calcula la diferencia ente los vectores en cuestión, de dos en dos, emplegando la correllación de Pearson, que mide l'asemeyu ente dos contornos como los que se representen na figura 11. 


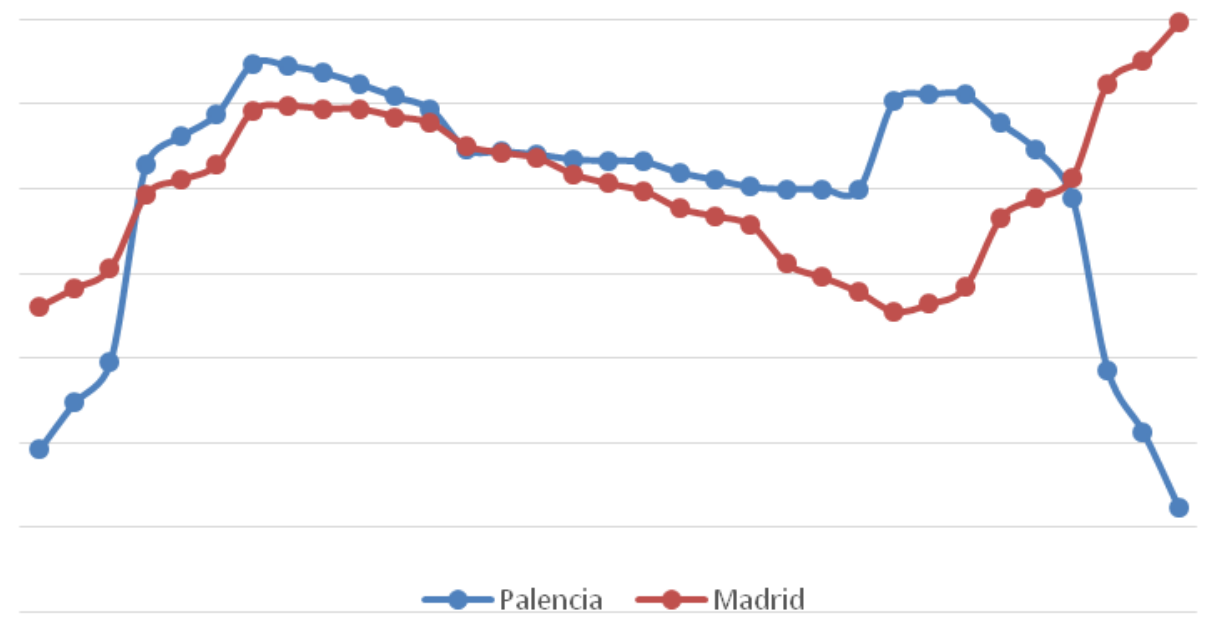

Figura 11. Representación gráfica de los vectores numbéricos xeneraos dende dos curves entonatives interrogatives (Palencia y Madrid, respeutivamente) que ProDis usa pa calcular la correllación de Pearson

La figura 12 representa l'aplicación de la fórmula de la correllación de Pearson a los datos prosódicos sacaos por Amper-2006. Na fórmula $f_{1}(i)$ ye'l valor de F0 en semitonos nel puntu $i$, mentanto que $m_{1}$ y $m_{2}$ son los valores medios de cada contornu. El programa ufre la posibilidá de calcular la correllación de Pearson namái a partir de los valores de F0, o bien de ponderalos pola intensidá, pola duración o por dambes $\left(w_{I}\right.$ ye'l coeficiente de ponderación que s'algama a partir de la media de los valores d'enerxía en dB y $w_{D}$ ye'l coeficiente de ponderación pola duración de la vocal en ms).

$$
R_{f_{1}, f_{2}}=\frac{\sum_{i} w_{I}(i) w_{D}(i)\left(f_{1}(i)-m_{1}\right)\left(f_{2}(i)-m_{2}\right)}{\sqrt{\sum_{i} w_{I}(i) w_{D}(i)\left(f_{1}(i)-m_{1}\right)^{2} \sum_{i} w_{I}(i) w_{D}(i)\left(f_{2}(i)-m_{2}\right)^{2}}}
$$

Figura 12. Fórmula de la correllación de Pearson aplicada a los datos prosódicos sacaos con Amper-2006

Nel corpus AMPER cada informante pronuncia tres veces la mesma frase, col envís de neutralizar posibles efeutos de pequeñes variaciones intrainformante. Poro, la correllación media $(\mathrm{CM})$ de cada enunciáu calcúlase 
considerando toles combinaciones posibles de les tres repeticiones de los dos informantes (pa un total de nueve permutaciones), y promediando los resultaos del cálculu de la correllación de Pearson. La CM calcúlase pa cada pareya d'informantes de la base de datos, lo qu'empobina a poder ufiertar una matriz por informantes (MI), que contién les correllaciones medies de cada informante con tolos demás. Nun segundu momentu, los informantes d'un mesmu puntu d'encuesta axúntense y algámase una matriz per puntos d'encuesta (MPE), que ye la qu'indica los asemeyos prosódicos qu'existen ente dos puntos d'encuesta d'AMPER.

Los valores numbéricos conteníos na MPE puen resultar d'interpretación abegosa. Por cuenta d'ello, ProDis camuda la MPE nun heatmap, ye a dicise, nuna matriz na que les destremaes gradaciones de color representen niveles de correllación distintos: los colores cálidos/escuros indiquen una correllación baxa, mentanto que los colores fríos/claros indiquen una correllación fuerte (nel apartáu de resultaos ufriránse dellos exemplos de heatmap de correllaciones).

A partir de la MPE, ProDis fai análisis que tienen l'oxetivu de visualizar les agrupaciones de puntos d'encuesta en base al asemeyu prosódicu ente ellos. La clas d'análisis al que se someten los datos prosódicos ye'l cluster analysis o análisis de conglomeraos, con resultaos representaos per aciu de diagrames d'escalamientu multidimensional (EMD) y dendrogrames. Lo mesmo pal casu de los mapes EMD que pal de los dendrogrames, ProDis ufre una estimación del grau de fiabilidá de les representaciones.

Pal casu de los mapes EMD, calcula un valor de stress (que apaez nel pie de la mesma figura, como por exemplu na figura 15 más alantre), que ye direutamente proporcional al grau de correspondencia ente la representación gráfica y les distancies reales ente los puntos: contra más baxu sía'l valor de stress, más fiable ye la representación en forma de mapa.

Nel casu de los dendrogrames, ProDis calcula'l númberu meyor d'agrupaciones a partir de los valores de silueta (Rousseeuw 1987). Nun gráficu como'l de la figura 13, el programa ufre información sobro los valores de silueta globales que s'algamen pa caún de los posibles númberos de clusters. Arriendes, pa mayor comodidá del investigador, conseña claramente (y marca con una flecha) el valor de silueta que correspuende al númberu meyor -dende'l puntu de vista estadísticu- de clusters (pa más detalles, ver Elvira-García 2017). Más abaxo, cuando s'apurran los resultaos del análisis de los datos del asturianu, presentaránse y discutiránse dellos exemplos de caún d'estos análisis, poniendo especial procuru na importancia de los valores del gráficu de silueta pa una interpretación acionada de los resultaos qu'ufierta'l cluster analysis. 


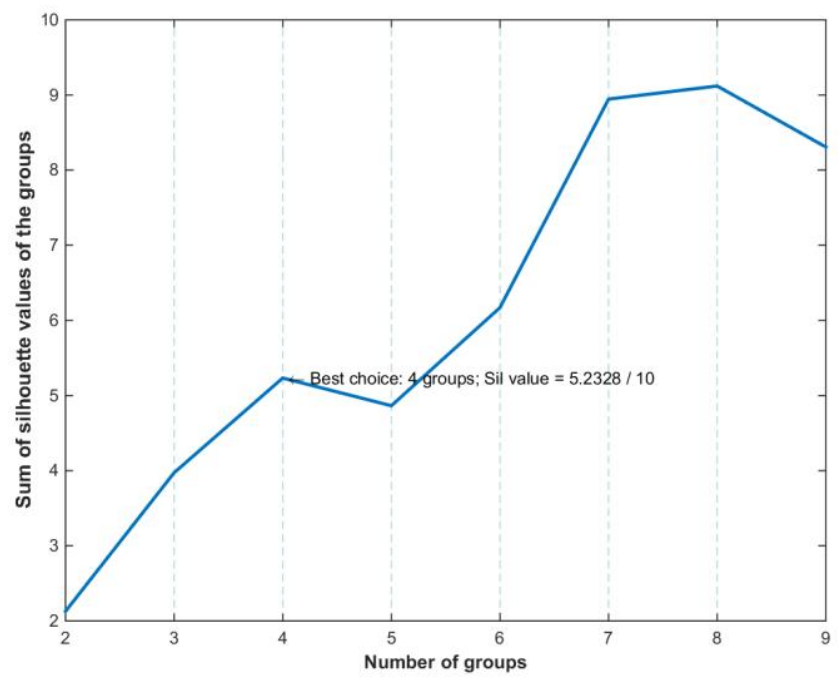

Figura 13. Exemplu de gráficu de valores globales de silueta xeneráu por ProDis. Pue alvertise que s'indica con una flecha'l númberu óptimu de clusters y el correspondiente valor global de silueta (Best choice: 4 groups; Sil. value $=5.2328 / 10$ )

\section{RESULTAOS}

Nos tres subapartaos d'esta seición preséntense los resultaos de tres análisis dialeutométricos fechos colos datos que conformen el corpus emplegáu. En primer llugar, tómense en consideración namái los enunciaos declarativos. Darréu úfrese un resume de los resultaos algamaos analizando les interrogatives. A lo cabero, sométense a análisis les dos modalidaes oracionales conxuntamente.

\section{Análisis dialeutométricu de les declaratives}

Si se lleven a un análisis dialeutométricu per aciu de ProDis les 1.890 declaratives neutres incluyíes nel corpus emplegáu pa esti trabayu, los resultaos que s'algamen nun paecen confirmar - polo menos a primer vista- lo que se defendió nos estudios anteriores sobro la prosodia del asturianu, que coinciden en describir un únicu patrón pa les declaratives neutres d'esa llingua, con variaciones locales perpoco pronunciaes. El heatmap de correllaciones (figura 14), de fechu, paez suxerir la existencia de dos bloques xeoprósodicos claros, (el primeru d'ellos conformáu por Uviéu y Mieres; el segundu, por Tinéu y El Franco, a los que s'amiesta Ribeseya). El mapa EMD (figura 15) confirma esa xuntura, lo mesmo que'l dendrograma (figura 16). A 
partir d'esos análisis, entós, tendría que se concluyir que dende'l puntu de vista de la prosodia de les declaratives, el dominiu llingüísticu asturianu xébrase nuna zona central (Uviéu y Mieres) a la que s'oponen les fasteres llaterales (la occidental con Tinéu y El Franco y la zona oriental con Ribeseya). Esa conclusión paez tar encontada pol valor mui baxu de stress del mapa EMD (indicáu na mesma figura 15), que suxer que la distribución de los puntos nel espaciu virtual ye estadísticamente recia.

Pero, con to y con ello, esa interpretación de los resultaos estadísticos nun tien en cuenta otru datu d'importancia cimera: si observamos los valores del gráficu de silueta (figura 17), ye perclaro que, dende'l puntu de vista estadísticu, la meyor solución de xuntura ye la qu'acaba clasificando tolos puntos d'encuesta nun mesmu conglomeráu. N'otres palabres, si bien ye cierto que l'algoritmu de cluster analysis pue estremar dos grupos xeoprosódicos a partir de los datos de les declaratives, la solución estadística meyor ye nun xebrar el dominiu llingüísticu asturianu. Eso coincide dafechu colo afitao polos trabayos anteriores (Muñiz Cachón et al. 2010; Alvarellos Pedrero et al. 2011; Muñiz Cachón 2013) que, a partir d'un análisis cualitativu y llingüísticu de los datos, afirmen que les dixebres ente los patrones de les declaratives son mínimos y nun dexen afitar clasificaciones. Nesti sen, podemos afirmar que l'análisis fechu polos llingüistes ta validáu dialeutométricamente.

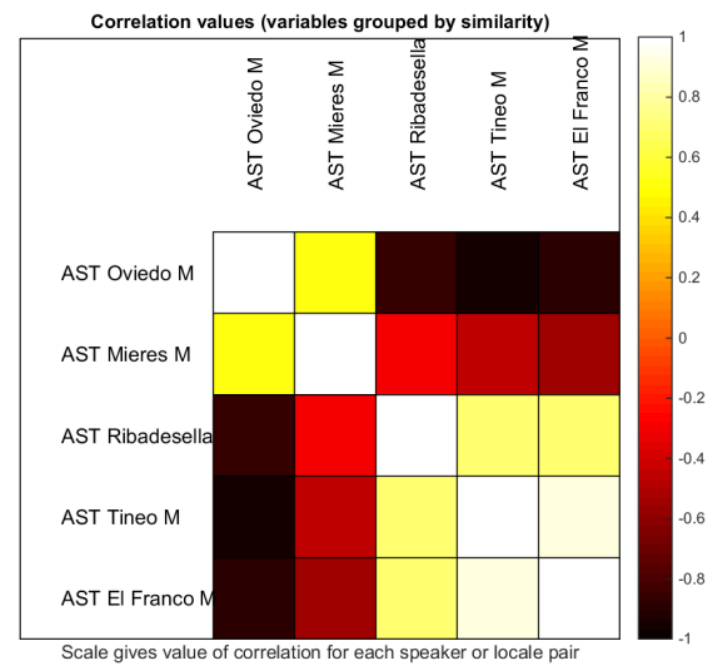

Figura 14. Heatmap de correllaciones ente los puntos d'encuesta a partir de los datos de F0 (ponderaos por duración ya intensidá) de les declaratives 


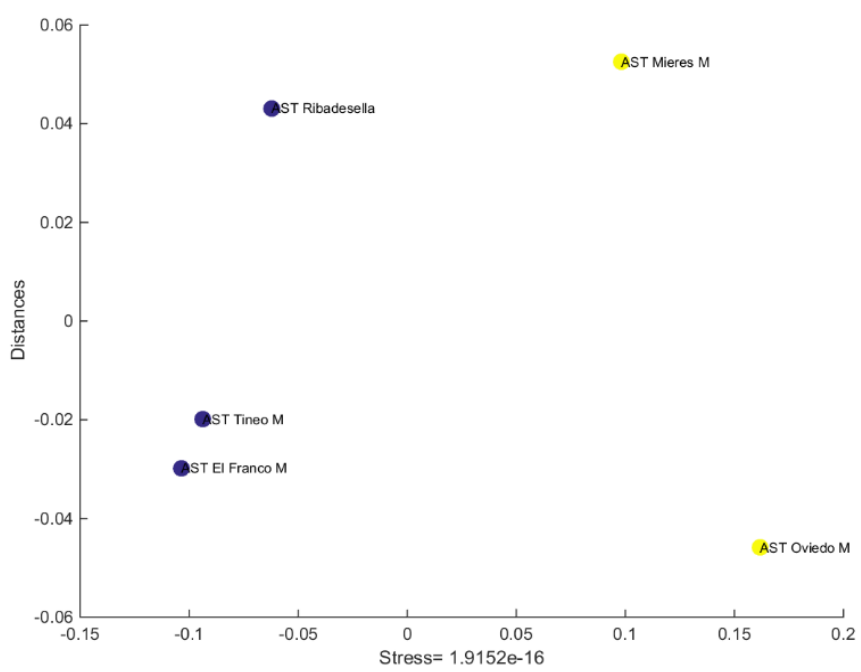

Figura 15. Mapa EMD de los puntos d'encuesta a partir de los datos de F0 (ponderaos por duración ya intensidá) de les declaratives

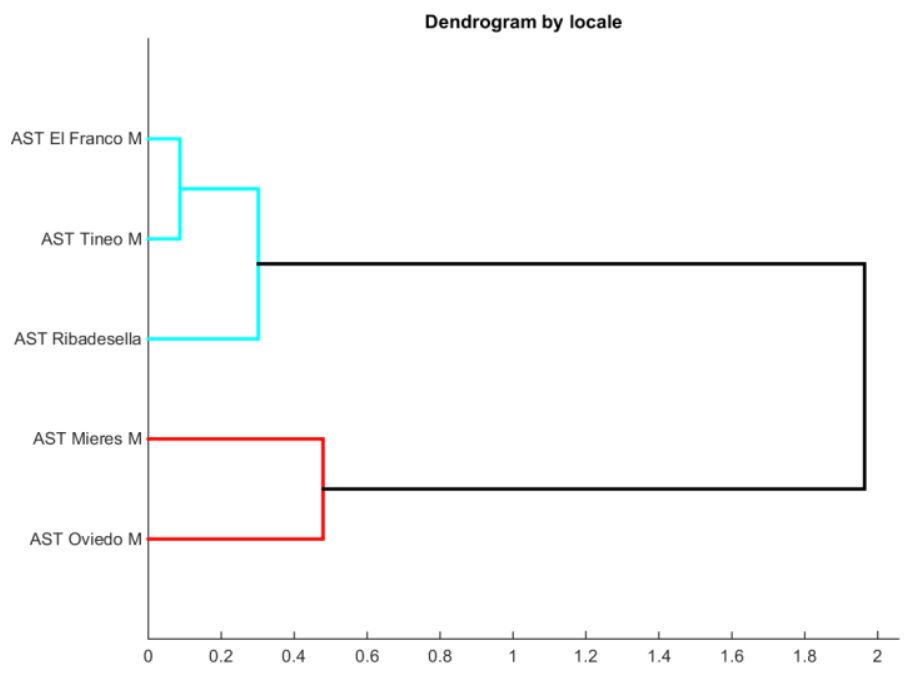

Figura 16. Dendrograma de los puntos d'encuesta a partir de los datos de F0 (ponderaos por duración ya intensidá) de les declaratives 


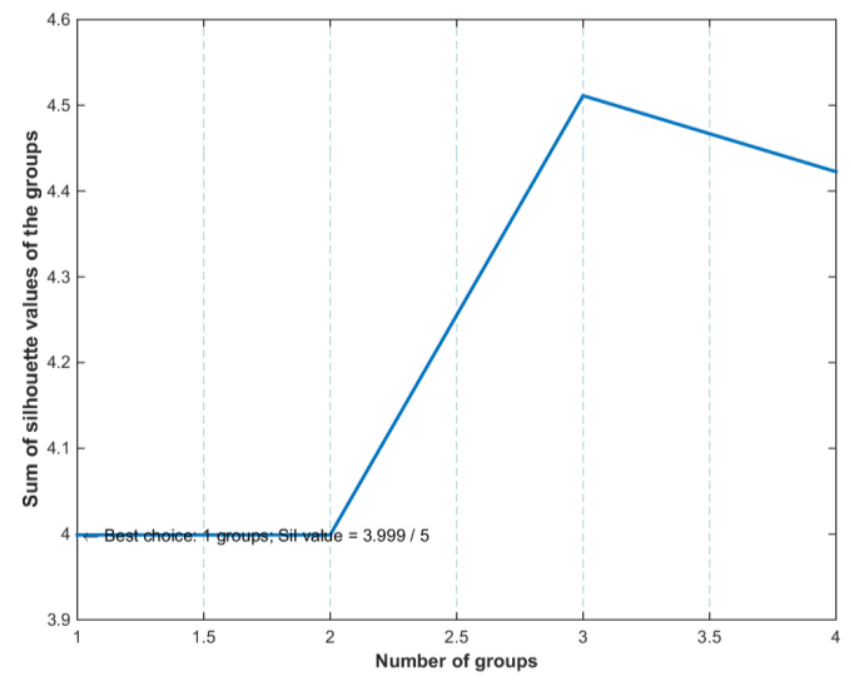

Figura 17. Gráficu de sumes de valores de silueta de los diferentes aglomeraos posibles de puntos d'encuesta a partir de los datos de F0 (ponderaos por duración ya intensidá) de les declaratives

\section{Análisis dialeutométricu de les interrogatives}

L'análisis dialeutométricu de les 1.890 interrogatives ufre resultaos destremaos, nel sen de que la existencia de dos bloques xeoprosódicos confírmase en tolos niveles. Los agrupamientos en cuestión, como se despriende del heatmap de correllaciones (figura 18), del mapa EMD (figura 19) y del dendrograma (figura 20), marquen una bipartición del dominiu llingüísticu asturianu, que queda xebráu nuna zona mayoritaria (qu'abarca El Franco, Tinéu, Uviéu y Mieres) y una zona oriental más finxada (qu'inxer namái a Ribeseya). La bipartición en cuestión resulta ser la meyor opción dende'l puntu de vista estadísticu, yá qu'asina lo amuesen los valores de silueta (figura 21).

Nel casu de la dialeutometrización de les interrogatives, ProDis ufre resultaos que namái en parte coinciden colo qu'afirmen estudios anteriores que s'afiten nuna comparanza cualitativa y llingüística de les curves entonatives. Recordemos que los trabayos sobro l'asturianu suxeren la presencia de cuatro zones xeoprosódiques en relación coles interrogatives: 1) la fastera occidental (El Franco y Tinéu) carauterizada polo que n'inglés se denoma hat pattern; 2) la fastera central (Uviéu) onde apaez un contornu descendente con dos picos entonativos suaves; 3) la fastera centromeridional (Mieres) na que'l patrón sigue presentando dos picos enforma más marcaos, pero na que la duración desempeña un papel cimeru y, a lo cabero, 4) la fastera oriental (Ribeseya) onde l'esquema entonativu nun ye propiamente descendente sinón qu'acaba con un movimientu de xubida y baxada dientro de la gama alta del informante. L'análisis 
dialeutométricu nun paez ser a estremar ente los primeros cuatro tipos de patrones interrogativos, que s'axunten nun mesmu cluster.

Nel casu del patrón de Mieres, la diverxencia ente l'análisis dialeutométricu y l'análisis fechu por llingüistes tien una xustificación acústica nidia: porque ProDis foi concibíu p'analizar un fenómenu prosódicu en concreto (ye a dicise, la entonación) y como la duración (que ye l'elementu más carauterísticu del patrón de Mieres) entra en xuegu namái como factor pel que se ponderen los valores de F0, ye claro que ProDis acaba deteutando -correutamente- un asemeyu perimportante ente les interrogatives d'Uviéu (figura 4) y de Mieres (figura 5).

El fechu de que ProDis axunte nun únicu cluster tolos patrones descendentes, ensin qu'importe que nel cuerpu presenten dos picos (como n'Uviéu y Mieres, figures 4 y 5, respeutivamente) o un plateau (como n'El Franco y Tinéu, figures 2 y 3 , respeutivamente), ye, a primer vista, más problemáticu, yá que pa un llingüista la dixebra ente los dos esquemes ye enforma evidente. Entá asina, la clasificación de ProDis pue llegar a entendese si se fai acordanza de cuála ye una de les carauterístiques principales de les téuniques de cluster analysis. Dende'l puntu de vista estadísticu, hai que tener en cuenta qu'una de les llendes d'estes téuniques ye qu'avecen dar resultaos bonos namás si'l númberu de suxetos ye lo bastante altu. Nos casos como esti qu'equí nos ocupa, onde namái hai cinco puntos d'encuesta (aunque en caún d'ellos haya dos informantes y cientos d'enunciaos analizaos), los resultaos puen nun ser los meyores. Sobro esti conceutu volveremos nel apartáu dedicáu al discutiniu de los resultaos.

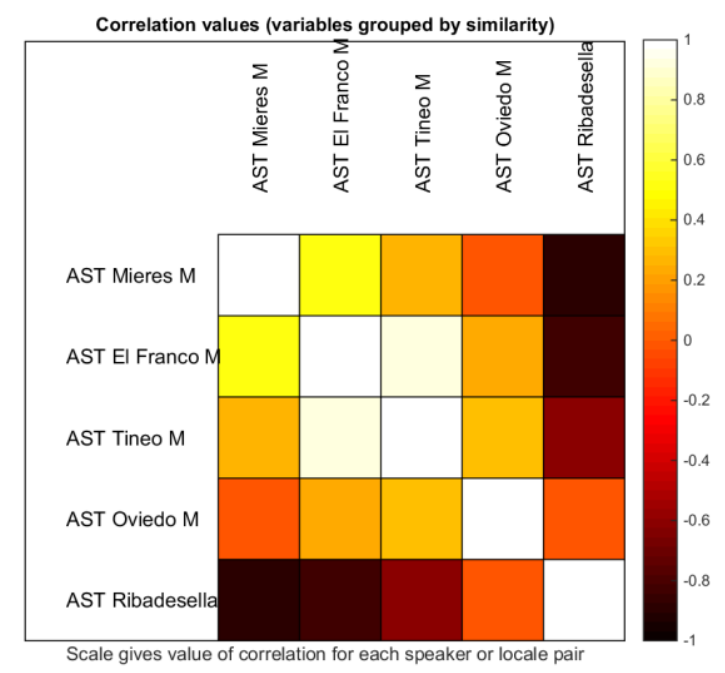

Figura 18. Heatmap de correllaciones ente los puntos d'encuesta a partir de los datos de F0 (ponderaos por duración ya intensidá) de les interrogatives 


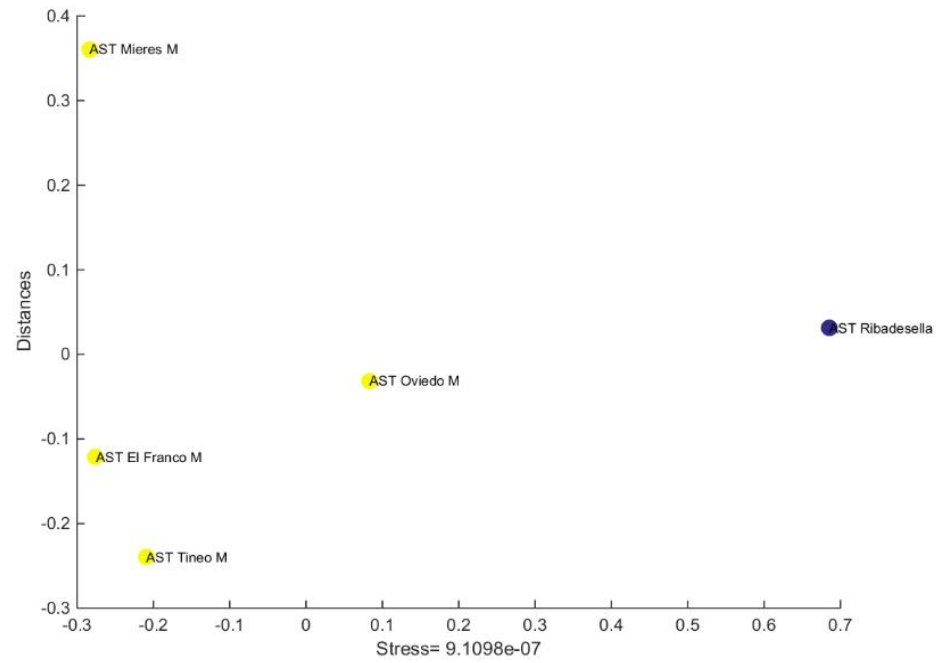

Figura 19. Mapa EMD de los puntos d'encuesta a partir de los datos de F0 (ponderaos por duración ya intensidá) de les interrogatives

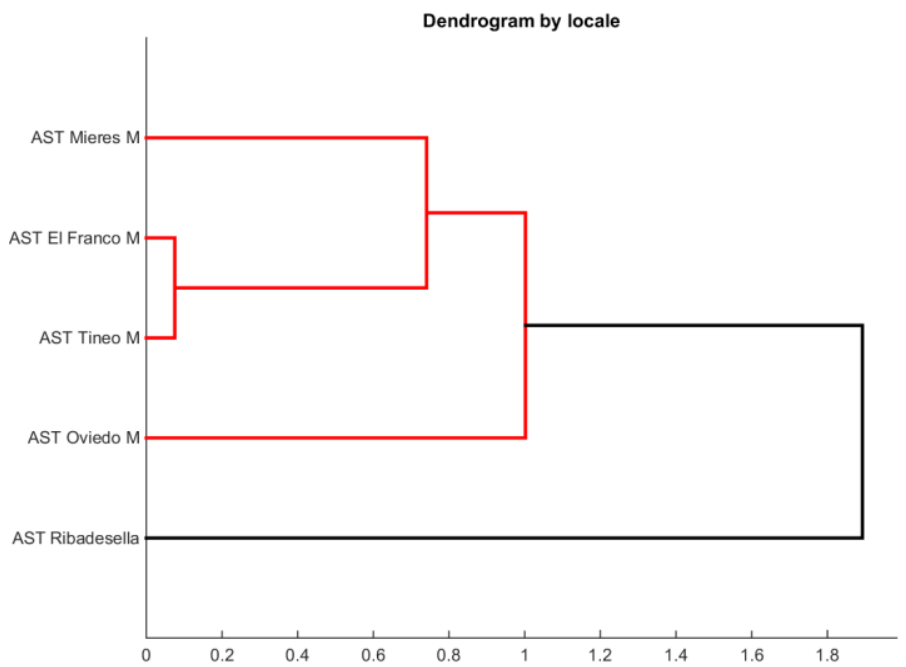

Figura 20. Dendrograma de los puntos d'encuesta a partir de los datos de F0 (ponderaos por duración ya intensidá) de les interrogatives 


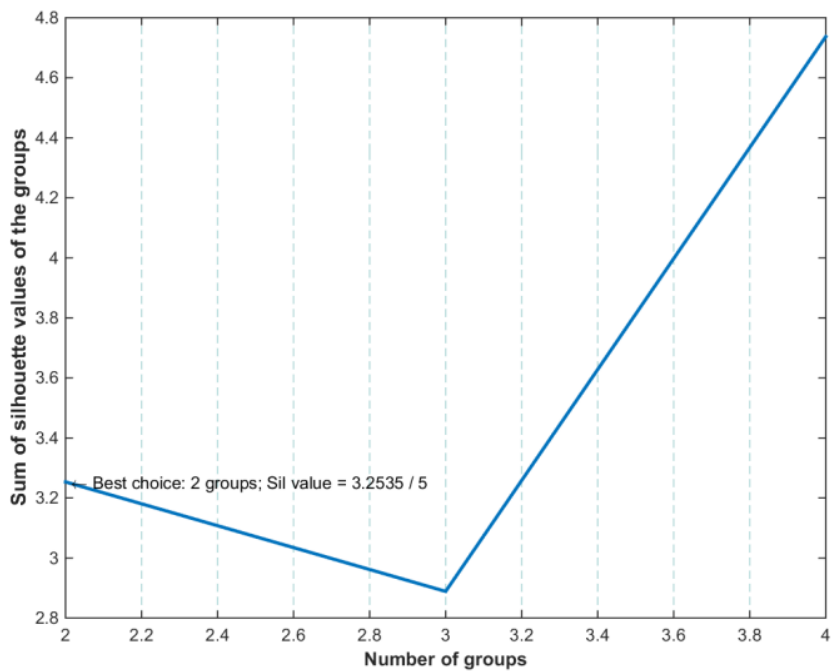

Figura 21. Gráficu de sumes de valores de silueta de los diferentes posibles aglomeraos de puntos d'encuesta a partir de los datos de F0 (ponderaos por duración ya intensidá) de les interrogatives

\section{Análisis dialeutométricu de declaratives ya interrogatives}

L'análisis conxuntu de les dos modalidaes oracionales confirma la bipartición que se describió nel apartáu anterior: l'Oriente d'Asturies (Ribeseya) xébrase de mou claru de la parte central y occidental (figures 22, 23 y 24). Si tenemos en cuenta los valores de los gráficos de silueta (figura 25), esa xebradura en dos bloques xeoprosódicos ye la meyor dende'l puntu de vista estadísticu.

El fechu de que los resultaos del análisis conxuntu de les dos modalidaes coincidan colos de les interrogatives ye un fenómenu que yá se tien visto n'otros casos (Fernández Planas et al. 2011, 2015a, 2015b, 2017; Roseano et al. 2015; ver Frota y Prieto 2015 pa un discutiniu). El casu d'estudiu del asturianu, poro, confirma un enclín alvertíu yá nes llingües romániques: les declaratives nun paecen contribuir a la definición de zones xeoprosódiques, mentanto que les interrogatives dexen estremar ente dialeutos. D'esi enclín o tendencia xeneral escápense bien poques llingües -como'l friulán (Roseano y Fernández Planas 2019)-, pa les que les isogloses entonatives más destacaes puen trazase a partir del contornu de les declaratives. 


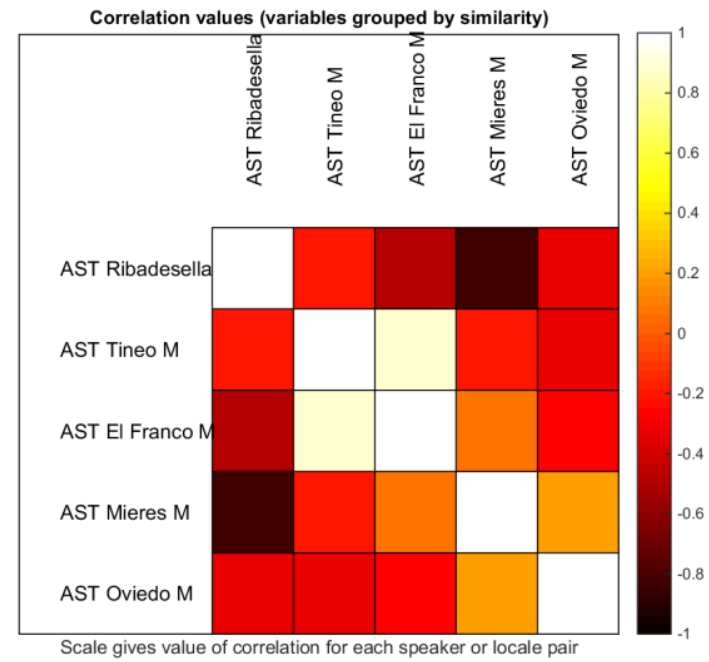

Figura 22. Heatmap de correllaciones ente los puntos d'encuesta a partir de los datos de F0 (ponderaos por duración ya intensidá) de les declaratives ya interrogatives

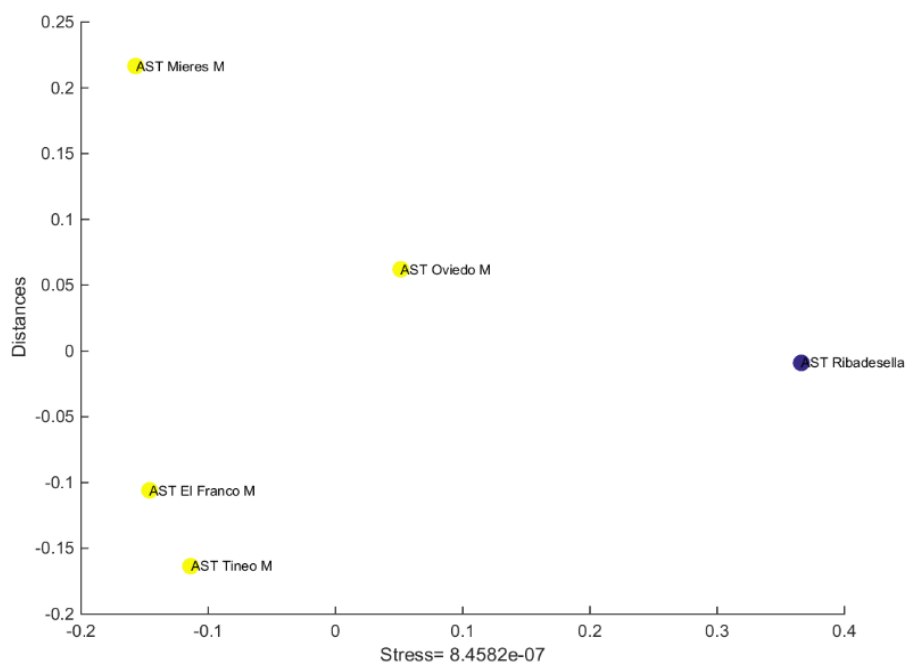

Figura 23. Mapa EMD de los puntos d'encuesta a partir de los datos de F0 (ponderaos por duración ya intensidá) de les declaratives ya interrogatives 


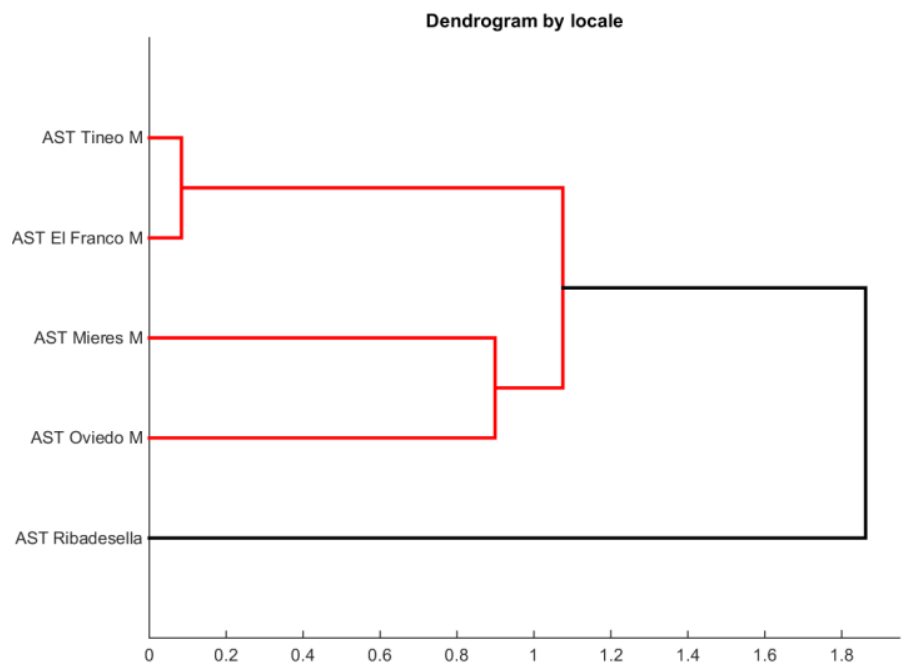

Figura 24. Dendrograma de los puntos d'encuesta a partir de los datos de F0 (ponderaos por duración ya intensidá) de les declaratives ya interrogatives

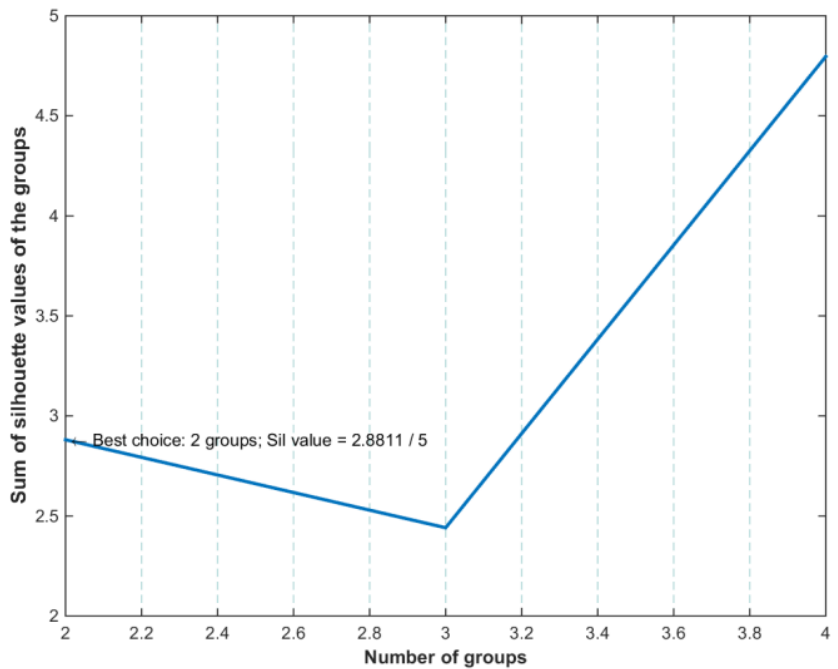

Figura 25. Gráficu de sumes de valores de silueta de los diferentes posibles aglomeraos de puntos d'encuesta a partir de los datos de F0 (ponderaos por duración ya intensidá) de les declaratives ya interrogatives 


\section{DISCUTINIU Y CONCLUSIONES}

L'análisis dialeutométricu de los datos d'AMPER-ASTUR ufierta delles conclusiones clares y, al empar, plantega unes entrugues de triba metodolóxica y dialeutolóxica (estes caberes lo mesmo en sincronía que'en diacronía).

El primer resultáu consiste na constatación de que la entonación de les declaratives nun permite dibuxar dixebres prosódiques clares ente los distintos puntos d'encuesta d'Asturies, tal y como yá conseñaren estudios anteriores. Nesti sen, l'asturianu nun se separta de lo que yá alvertío na mayoría de llingües romániques.

D'otra banda, les interrogatives ufren elementos abondos pa llegar a xebrar el dominiu llingüísticu asturianu en, polo menos, dos bloques xeoprosódicos. El primeru d'ellos (El Franco, Tinéu, Uviéu y Mieres) ta carauterizáu por interrogativos totales descendentes, mentes que'l segundu (Ribeseya) presenta un patrón interrogativu carauterizáu por un plateau altu qu'acaba con un movimientu circunflexu de xubida y baxada. Tal y como vimos enantes, esa bipartición ye una clasificación muncho menos iguada y peñerada que la que propón la lliteratura. Sorpriende de mou particular que ProDis nun sía quien, aparentemente, pa estremar ente los patrones occidentales (carauterizaos por un hat pattern) y los del centru d'Asturies (que presenten dos picos nel cuerpu de les interrogatives).

La incapacidá de ProDis de deteutar dixebres entonatives que nun sían macroscópiques ye, en realidá, namái aparente y tien una esplicación epistemolóxica perlóxica. Hai que decatase de que ProDis sofitase n'algoritmos de cluster analysis, que tán pensaos p'amenorgar -cueste lo que cueste- el númberu de suxetos per aciu d'aglomeraciones. De fechu, les téuniques de cluster analysis nacieron pa bregar col enzancu que representaba'l fechu de tener que xestionar un númberu altu de suxetos. Dicho d'otra miente, tán pensaes pa funcionar bien nun contestu onde se dea un númberu altu de suxetos que clasificar y van agrupalos inclusive a costa de perder información. Nos casos como'l qu'agora nos ocupa, onde'l númberu de suxetos (pal nuestru casu de puntos d'encuesta) ye llendáu, los resultaos puen resultar forzaos (Formann 1984; Dolnicar 2002) porque los algoritmos tán pensaos p'axuntar: van crear clusters hasta nel casu de qu'aglomerar implique una perda d'información que va a la escontra del criteriu del investigador. Pa que la perda d'información debida al clustering sía acoyible y nun vaya a la escontra del conocimientu llingüísticu del investigador, fai falta disponer d'un númberu prestamosu de suxetos (pal casu nuesu, de puntos d'encuesta).

Les maneres pa poder disponer d'un númberu abondu de puntos d'encuesta pa un análisis dialeutométricu con ProDis son dos: d'una parte podría multiplicase'l númberu de puntos d'encuesta dientro d'Asturies; pero, anque ello paeza una opción vidable, nun sedría, o asina-y abulta a quien esto escribe, la solución meyor, porque significaría una pergrán cantidá de trabayu en rellación a 
los resultaos que podría ufiertar; d'otru llau, otra manera de llograr un númberu abondu de puntos d'encuesta consiste n'enanchar la llende xeográfica considerada y amestar puntos d'otres zones llingüístiques, los más averaos en primer llugar y llueu los más alloñaos.

Tal y como se suxirió apocayá, nun primer averamientu nesti sen (Roseano 2018), inxerir los datos del asturianu nun análisis que comprienda tamién puntos de la península ibérica (como Tanos ${ }^{2}$ en Cantabria, Palencia y Soria en Castiya y Lleón, Madrid nel castellanu central, València y Castelló de la Plana nel dominiu llingüísticu catalán), así como dellos puntos de Friul, non sólo permite situar al asturianu nel mapa prosódicu de la Romania, sinón que tamién pon lluz nuevo sobro la subdivisión interna del asturianu mesmu. Como se pue alvertir na figura 26, analizando los datos d'AMPER-ASTUR nun marcu más ampliu pue apreciase que la fastera oriental d'Asturies presenta un asemeyu prosódicu interesante con Cantabria occidental, pero tamién cola variedá, xeográficamente relativamente cercana, de Soria. D'otra miente, confírmase que Tinéu y El Franco amuesen patrones melódicos muncho asemeyaos. Uviéu, pela so parte, resulta tener un semeyante significativu col llugar castellanolleonés de Palencia (como yá s'afita en Fernández Planas et al. 2017), mentanto que Mieres asítiase nuna posición claramente más alloñada dientro del mesmu bloque.

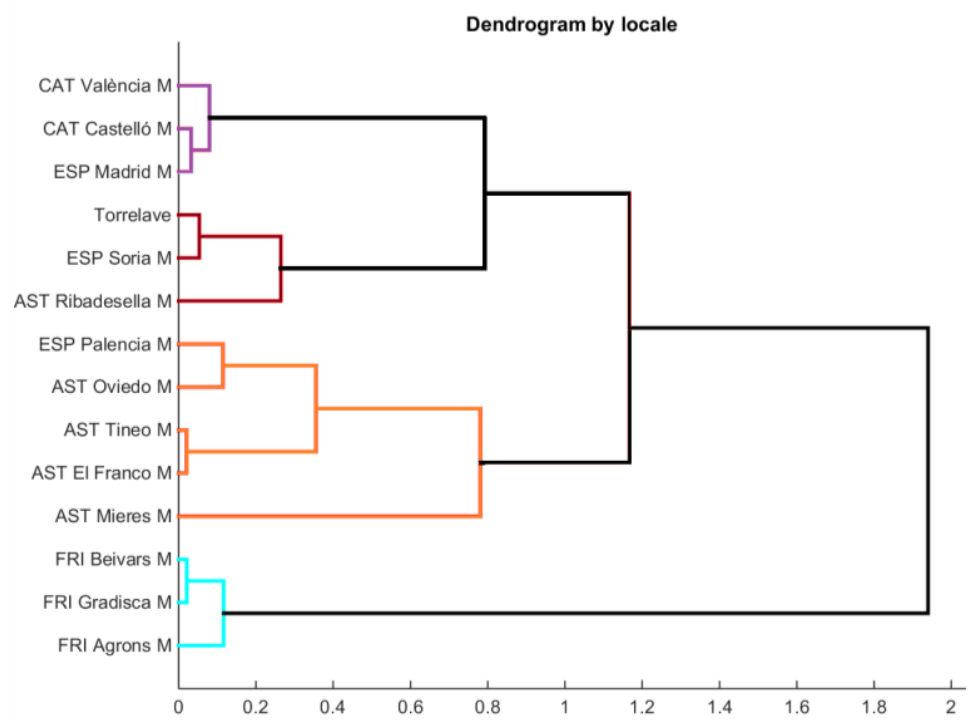

Figura 26. Dendrograma de los puntos d'encuesta a partir de los datos de F0 (ponderaos per duración ya intensidá) de les declaratives ya interrogatives ensin espansión de dellos puntos de los dominios llingüísticos asturianu, friulanu, catalán y español peninsular (fonte: Roseano 2018)

${ }^{2}$ Al tratase d'un puntu d'encuesta fuera de los dominios d'AMPER-ASTUR y d'AMPERCAT, namái se grabó una muestra restrinxida d'enunciaos pa un estudiu esploratoriu. 
Poniendo puntu yá, esta primer aplicación de les téuniques d'análisis dialeutométricu a los datos d'AMPER-ASTUR confirma, en llinies xenerales, los estudios previos. Sicasí, suxer al empar que la meyor manera pa llegar a una comprensión axeitada de la prosodia del asturianu (tanto en rellación colos sos rasgos específicos como en rellación colos aspeutos que comparte con otres llingües romániques) ye ampliar la comparanza hasta llegar, polo menos idealmente, a analizar conxuntamente toles variedaes romániques de la península ibérica. Eso non sólo conformaría un avance dialeutolóxicu y xeoprosódicu en sincronía, sinón que tamién podría poner lluz sobro la complexa historia llingüística de la península, como yá propunxeron otros autores na cabera década (Dorta Luis y Muñiz Cachón 2009; Congosto et al. 2010; López Bobo et al. 2012; Bleortu y Cuevas Alonso 2014, 2015; Fernández Rei 2018; Fernández Planas et al. 2017).

\section{Agradecimientos}

Esti trabayu féxose en marcu del proyeutu Tecnologías derivadas de AMPER-CAT y análisis de corpus complementarios (FFI2015-64859-P), subvencionáu pol Ministeriu d'Economía y Competitividá, y sofítase nos resultaos del proyeutu Descripción y estudio de los modelos entonativos en las variedades lingüísticas de Asturias: el castellano de Asturias (FFI200803625/FILO), financiáu pol Ministeriu de Ciencia ya Innovación (MICINN). L'autor quiere agradecer la collaboración de la Dra. Carmen Muñiz Cachón, de la Dra. Ana María Fernández Planas y de la Dra. Wendy Elvira-García, ensin les qu'esti estudiu nun sedría posible, asina como la de l'Academia de la Llingua Asturiana, que s'encargó de la traducción al asturianu del manuscritu orixinal.

\section{REFERENCIES BIBLIOGRÁFIQUES}

Alvarellos Pedrero, Mercedes \& Carmen MuñIz Cachón \& Liliana Díaz Gómez \& Ruth GONZÁLEZ RODRíGUEZ (2011): «La entonación en las variedades lingüísticas de Asturias: estudio contrastivo», en RILI 17: 111-120.

AurreKoetxea, Gotzon \& Karmele Fernandez-Aguirre \& Jesús Rubio \& Borja Ruiz \& Jon SÁNCHEZ (2013): «DiaTech: A new tool for dialectology», en Literary and Linguistic Computing 28(1): 23-30.

Bleorțu, Cristina \& Miguel Cuevas Alonso (2014): «La entonación de las variedades norteñas y la existencia de un continuum panrománico», Vi Congreso Internacional de Fonética Experimental, Universitat de València, València, 4-8/10/2014.

- (2015): «Las variedades entonativas cántabras en el continuum norteño», IV Jornadas de Lingüística Hispánica, Universidade de Lisboa, Lisboa, 21-22/4/2015.

Congosto Martín, Yolanda \& Liliana Díaz Gómez \& María Viejo Lucio-Villegas \& Ruth FERNÁNDEZ RODRÍGUEZ (2010): «Estudio contrastivo de la entonación del castellano de 
Don Benito y del asturiano de Mieres en el marco del Proyecto AMPER», en Actes $d u$ XXVe CILPR Congrès International de Linguistique et de Philologie Romanes. Maria Iliescu \& Heidi Siller-Runggaldier \& Paul Danler (eds.). Berlin/New York, De Gruyter: 253-266.

Contini, Michel \& Jean-Pierre Lai \& Antonio Romano \& Stephanie Roullet (2003): «Vers un Atlas prosodique des variétés romanes», en Sempre los camps auràn segadas resurgantas, Mélanges offerts a Xavier Ravier. Jean-Claude Bouvier \& Jacques Gourc \& François Pic (eds.). Toulouse, CNRS: 73-84.

CONTINI, Michel (1992): «Vers une géoprosodie romane», en Nazioarteko dialektologia biltzarra: Agiriak. Gotzon Aurrekoetxea \& Xarles Videgain (eds.). Bilbo, Euskaltzaindia: 83-109.

DOLNICAR, Sara (2002): «A review of unquestioned standards in using cluster analysis for datadriven market segmentation». University of Wollongong Faculty of Commerce-Papers, 273.

DorTa Luis, Josefa \& María del Carmen MuÑIz CACHón (2009): «La entonación de las interrogativas en el español de Canarias y en asturiano», n'Homenaje al Profesor Dr. Wolfredo Wildpret de la Torre. Esperanza Beltrán Tejera \& Julio Alfonso Carrillo \& Antonio García Gallo \& Octavio Rodríguez Delgado (eds.). La Laguna, Instituto de Estudios Canarios: 809-821.

Elvira-García, Wendy \& Simone Balocco \& Paolo Roseano \& Ana Ma Fernández Planas (2018): «ProDis: A dialectometric tool for acoustic prosodic data», en Speech Communication 97: 9-18.

ELVIRA-GARCíA, Wendy (2017): "L'índex del gràfic de silueta com a eina com a eina metodològica per avaluar l'optimitat dels clústers dialectals», IX Workshop de l'Entonació del Català, Universitat Pompeu Fabra, Barcelona, 4/7/2017.

Fernández Planas, Ana $M^{\mathrm{a}}$ \& Paolo Roseano \& Eugenio Martínez Celdrán \& Lourdes ROMERA BARRIOS (2011): «Aproximación al análisis dialectométrico de la entonación en algunos puntos del dominio lingüístico catalán», n'Estudios de Fonética Experimental 20: 141-178.

Fernández Planas, Ana Ma \& Josefa Dorta \& Paolo Roseano \& Xaxiraxi Díaz \& Wendy Elvira-García \& José Antonio Martín Gómez \& Eugenio MARTínez CELdRÁn (2015a): «Distancia y proximidad prosódica entre algunas variedades del español: un estudio dialectométrico a partir de datos acústicos», en Revista de Lingüística Teórica y Aplicada 53(2): 13-45.

Fernández Planas, Ana $M^{\mathrm{a}} \&$ Paolo Roseano \& Wendy Elvira-García \& Ramon CerdàMassó \& Lourdes Romera Barrios \& Josefina Carrera-SAbaté \& Dorota SzmidT \& Sabela LABRAÑa \& Eugenio MARTínez CELDRÁN (2015b): «Cap a un nou mapa dialectal del català? Consideracions a partir de dades prosòdiques tractades dialectomètricament», n'Estudios de Fonética Experimental 24: 257-286.

Fernández Planas, Ana M ${ }^{\mathrm{a}}$ \& Josefa Dorta Luis \& Carmen MuñIz Cachón \& Paolo Roseano \& Wendy Elvira-García \& Ramon Cerdà Massó (2017): «La prosodia palentina en el Atlas Multimedia de Prosodia del Espacio Románico en España», n'Anadiss hors série: 61-76.

FERnÁNDEZ Rei, Elisa \& Lourdes DE CASTRo Moutinho (2016): «Estudo xeolingüístico da entoación galego-portuguesa», en 53 reflexiones sobre aspectos de la fonética y otros temas de lingüística. Ana $\mathrm{M}^{\mathrm{a}}$ Fernández Planas (ed.). Barcelona, Laboratori de Fonètica de la Universitat de Barcelona: 201-208.

FERNÁNDEZ REI, Elisa (2016): «Dialectal, historical and sociolinguistic aspects of Galician intonation», en Dialectologia special Issue vi: 147-169.

- (2018): «El asturiano y las áreas geoprosódicas del occidente ibérico», XXXVII Xornaes Internacionales d'Estudiu, Academia de la Llingua Asturiana, Uviéu, 7/11/2018.

Formann, Anton K. (1984): Die Latent-Class-Analyse: Einfuhrung in die Theorie und Anwendung. Beltz, Weinheim. 
Frota, Sónia \& Pilar PriEto (2015): «Intonation in Romance: Systemic similarities and differences», n'Intonation in Romance. Sónia Frota \& Pilar Prieto (eds.). Oxford, Oxford University Press: $392-418$.

GoEbL, Hans (1982): Dialektometrie: Prinzipien und Methoden des Einsatzes der numerischen Taxonomie im Bereich der Dialektgeographie. Wien, Verlag der Österreichischen Akademie der Wissenschaften.

HAIMERL, Edgar (2006): «Database design and technical solutions for the management, calculation, and visualization of dialect mass data», en Literary \& Linguist Computing 21(4): 437-444.

HERMES, Dik J. (1998a): «Auditory and visual similarity of pitch contours», en Journal of Speech, Language and Hearing Research 41: 63-72.

- (1998b): «Measuring the perceptual similarity of pitch contours», en Journal of Speech, Language and Hearing Research 41: 73-82.

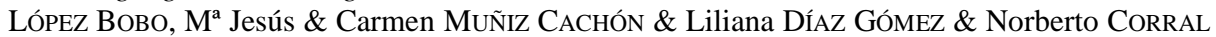
Blanco \& David Brezmes Alonso \& Mercedes Alvarellos Pedrero (2007): «Análisis y representación de la entonación. Replanteamiento metodológico en el marco del proyecto AMPER», en La prosodia en el ámbito lingüístico románico. Josefa Dorta (ed.). Santa Cruz de Tenerife, La Página: 17-34.

López Bobo, Ma Jesús \& Miguel Cuevas Alonso \& Álvaro Arias Cabal \& Cristina BleorȚu (2012): «El patrón entonativo norteño. Evidencia del español hablado en Galicia, Asturias y Cantabria», en Language and Literature: European Landmarks and Identity 11(2): 434440.

LóPez Bobo, Ma Jesús \& Miguel Cuevas Alonso \& Liliana Díaz Gómez \& María Viejo LuCiO-VILlegas (2008): «Prosodia del occidente de Asturias: secuencias con estructura sujeto-verbo-objeto», n'Archivum LVII: 1-32.

LóPez Bobo, $\mathrm{M}^{\mathrm{a}}$ Jesús \& Ruth GonzÁlez Rodríguez \& Miguel Cuevas Alonso \& Liliana DíAz GómeZ \& Carmen MuÑIZ CACHÓN (2005): «Rasgos prosódicos del centro de Asturias: comparación Oviedo-Mieres», n'Estudios de Fonética Experimental XIV: 167199.

Martínez CAlvo, Adela \& Elisa Fernández Rei (2015): «Unha ferramenta informática para a análise dialectométrica da prosodia», n'Estudios de Fonética Experimental xxIV: 289-303.

Moutinho de CASTRo, Lurdes \& Rosa Coimbra Lidia \& Albert Rilliard \& Antonio Romano (2011): «Mesure de la variation prosodique diatopique en portugais européen», n'Estudios de Fonética Experimental xx: 33-56.

MuñIz Cachón, Carmen \& Liliana Díaz Gómez \& Mercedes Alvarellos Pedrero \& Ruth GonZÁlez RodrígueZ (2010): «La prosodia d'Asturies», n'Homenaxe al Profesor Xosé Lluis García Arias. Uviéu, Academia de la Llingua Asturiana: 279-315.

Muñiz CACHÓn, Carmen \& Mercedes Alvarellos Pedrero (2008): «Una nota peculiar en la prosodia asturiana: la entonación oriental», en La variation diatopique de l'intonation dans le domain roumain et roman. Adrian Turculeţ (ed.). Iaşi, Editura Universitatii «Alexandru Ioan Cuza»:103-122.

MuÑIz CACHÓN, Carmen \& Ruth GonZÁlez RodRÍGuEZ \& Liliana DíAz GómEZ \& Mercedes AlVARELLOS PEDRERO (2006-2007-2008): «Prosodia gallego-asturiana en enunciaos SVO», en Revista de Filoloxía Asturiana 6-7-8: 335-349.

MuÑIZ CACHÓN, Carmen (2013): «La entonación asturiana n’el marcu de les llingües romániques: los atles posódicos», en Lletres Asturianes 109: 11-29.

Muñiz Cachón, Carmen (dir.) \& Mercedes Alvarellos Pedrero \& Liliana Díaz Gómez \& Ruth GonZÁlez RodrígueZ \& Llucía MenÉNdeZ SuÁrez (2019): AMPER-ASTUR. https://www.unioviedo.es/labofone/

Nerbonne, John \& Rinke Colen \& Charlotte Gooskens \& Peter Kleiweg \& Therese LEINONEN (2011): «Gabmap: A web application for dialectology», en Dialectologia special issue II: $65-89$. 
Rilliard, Albert \& Jean-Pierre LAI (2008): «La Base de Données AMPER et ses interfaces: structure et formats de données, exemple d'utilisation pour une analyse comparative de la prosodie de différents parlers romans», n'Actas das I Jornadas Científicas AMPERPOR. Lurdes Moutinho de Castro \& Rosa Lídia Coimbra (eds.). Aveiro, Universidade de Aveiro: 127-139.

RomAnO, Antonio \& Jean-Pierre LAI \& Stephanie Roullet (2005): «La méthodologie AMPER», en Géolinguistique hors serie 3, 1-5.

Romano, Antonio (1999): Analyse des structures prosodiques des dialectes et de l'italien régional parlés dans le Salento (Italie): Approche linguistique et instrumental. Tesis doctoral, Université Stendhal Grenoble 3, Grenoble.

Roseano, Paolo \& Ana $\mathrm{M}^{\mathrm{a}}$ Fernández Planas \& Wendy Elvira-García \& Eugenio MARTíNEZ CELDRÁN (2015): «Contacto lingüístico y transferencia prosódica: el caso del alguerés», en Dialectologia et Geolinguistica 23: 95-123.

RoseAno, Paolo \& Ana M ${ }^{a}$ FernÁndez Planas (2019): «Anàlisi estadística de les distàncies entonatives: el cas del friülà i de l'italià regional de Friül», n'Estudis Romànics 41: 235266.

Roseano, Paolo (2016): «Dos décadas de dialectometría entonativa», en Linguistic Variation in the Basque Language and Education II. Aitor Iglesias, Asier Romero \& Ariane Ensunza (eds.), Bilbao, Universidad del País Vasco: 56-80.

- (2018): «Análisis dialectométrico de la prosodia del asturiano en el ámbito románico: una primera aproximación», XXXVII Xornaes Internacionales d'Estudiu, Academia de la Llingua Asturiana, Uviéu, 7/11/2018.

SEGUY, Jean (1971): «La dialectométrie dans l'Atlas linguistique de Gascogne», en Revue de Linguistique Romane 37: 1-4. 